\title{
A Priori Estimates of Solution of Parametrized Singularly Perturbed Problem
}

\author{
Mustafa Kudu1, Ilhame Amirali² \\ ${ }^{1}$ Department of Mathematics, Faculty of Arts and Sciences, Erzincan University, Erzincan, Turkey \\ ${ }^{2}$ Department of Mathematics, Faculty of Sciences, Duzce University, Duzce, Turkey \\ Email: muskud28@yahoo.com, ailhame@gmail.com
}

Received 10 September 2015; accepted 17 January 2016; published 20 January 2016

Copyright (C) 2016 by authors and Scientific Research Publishing Inc.

This work is licensed under the Creative Commons Attribution International License (CC BY).

http://creativecommons.org/licenses/by/4.0/

c) (i) Open Access

\section{Abstract}

In this paper, we consider a parameterized singularly perturbed second order quasilinear boundary value problem. Asymptotic estimates for the solution and its first and second derivatives have been established. The theoretical estimates have been justified by concrete example.

\section{Keywords}

Parameterized Problem, Asymptotic Bounds, Singular Perturbation, Boundary Layer

\section{Introduction}

In this paper, we are going to obtain the asymptotic bounds for the following parameterized singularly perturbed boundary value problem (BVP):

$$
\begin{gathered}
L u:=\varepsilon u^{\prime \prime}-a(t) u^{\prime}-f(t, u, \lambda)=0,0<t<T, \\
u(0)=\mu_{0}, \quad u^{\prime}(0)=\mu_{1}, u^{\prime}(T)=\frac{\mu_{2}}{\varepsilon}
\end{gathered}
$$

where $0<\varepsilon \leq 1$ is the perturbation parameter, $\mu_{i}(i=0,1,2)$ are given constants and $0<\alpha \leq a(t) \leq a^{*}$ is a sufficiently smooth function in $[0, T]$. Further, the function $f(t, u, \lambda)$ is assumed to be sufficiently continuously differentiable for our purpose function in $\{0 \leq t \leq T,-\infty<u, \lambda<\infty\}$ and

$$
0 \leq \frac{\partial f}{\partial u} \leq M_{1}^{*}, \quad 0<m_{1} \leq\left|\frac{\partial f}{\partial \lambda}\right| \leq M_{1}<\infty .
$$


By a solution of (1.1), (1.2), we mean pair $\{u(t), \lambda\} \in C^{1}[0, T] x \mathbb{R}$ for which problem (1.1), (1.2) is satisfied. An overview of some existence and uniqueness results and applications of parameterized equations may be obtained, for example, in [1]-[10]. In [11]-[14] have also been considered some approxi-mating aspects of this kind of problems. The qualitative analysis of singular perturbation situations has always been far from trivial because of the boundary layer behavior of the solution. In singular perturbation cases, problems depend on a small parameter $\varepsilon$ in such a way that the solution exhibits a multiscale character, i.e., there are thin transition layers where the solution varies rapidly while away from layers it behaves regularly and varies slowly [15]-[18]. In this note, we establish the boundary layer behaviour for $u(t)$ of the solution of (1.1)-(1.2) and its first and second derivatives. Example that agrees with the analytical results is given.

\section{The Continuous Problem}

Lemma 2.1. Let $a(t) \geq \alpha>0, b(t) \geq 0$ and $\Phi(t)$ be the continuous functions on $[0, T]$. Then, the solution of the boundary-value problem

$$
\begin{gathered}
L v:=\varepsilon v^{\prime \prime}-a(t) v^{\prime}-b(t) v=\Phi(t), 0<t<T, \\
v(0)=\mu_{0}, v^{\prime}(T)=\frac{\mu_{2}}{\varepsilon},
\end{gathered}
$$

satisfies the inequality

$$
|v(t)| \leq\left|\mu_{0}\right|+\left|\mu_{2}\right| \omega(t)+\alpha^{-1} t\|\Phi\|_{\infty}, 0 \leq t \leq T
$$

where

$$
\omega(t)=\alpha^{-1}\left(\mathrm{e}^{\frac{-\alpha(T-t)}{\varepsilon}}-\mathrm{e}^{\frac{-\alpha T}{\varepsilon}}\right) .
$$

Proof. Under the above conditions, the operatör $L v$ admits the folloving maximum principle:

Suppose $v(t) \in C^{2}[0, T]$ be any function satisfiying $L v \leq 0 \quad(0<t<T), \quad v(0) \geq 0$ and $v^{\prime}(T) \geq 0$. Then, $v(t) \geq 0$ for all $t \in[0, T]$.

Now, for the barrier fonction

$$
\Psi_{ \pm}= \pm v(t)+\left|\mu_{0}\right|+\left|\mu_{2}\right| \omega(t)+\alpha^{-1} t\|\Phi\|_{\infty},
$$

taking also into consideration that, $\omega(t)$ is a solution of the problem

$$
\varepsilon \omega^{\prime \prime}-\alpha \omega^{\prime}=0, \omega(0)=0, \omega^{\prime}(T)=\frac{1}{\varepsilon},
$$

it follows that,

$$
\begin{gathered}
L \Psi_{ \pm}= \pm \Phi(t)-b(t)\left|\mu_{0}\right|+\left|\mu_{2}\right|(\alpha-\alpha(t)) \omega^{\prime}(t) \\
-\left|\mu_{2}\right| b(t) \omega(t)-a(t) \alpha^{-1}\|\Phi\|_{\infty}-b(t) \alpha^{-1} t\|\Phi\|_{\infty} \\
\leq \pm \Phi(t)-a(t) \alpha^{-1}\|\Phi\|_{\infty} \leq 0, \\
\Psi \pm(0)= \pm \mu_{0}+\left|\mu_{0}\right| \geq 0 \\
\Psi^{\prime} \pm(T)= \pm \frac{\mu_{2}}{\varepsilon}+\frac{1}{\varepsilon}\left|\mu_{2}\right| \geq 0
\end{gathered}
$$

therefore $\Psi \pm(t) \geq 0$, which immediayely leads to (2.3).

Remark 1 . The inequality (2.3) yields.

$$
|u(t)| \leq\left|\mu_{0}\right|+\left|\mu_{2}\right| \omega(t)+\alpha^{-1} T\|\Phi\|_{\infty} .
$$

Theorem 2.1. For $\rho=1-\alpha^{-1} m_{1}^{-1} M_{1} M_{1}^{*} T$ and under conditions (1.3), the solution $\{u(t), \lambda\}$ of the problem 
(1.1), (1.2), satisfies,

$$
\begin{gathered}
|\lambda| \leq c_{0} \\
|u| \leq c_{1}
\end{gathered}
$$

where

$$
\begin{gathered}
c_{0}=\rho^{-1}\left[\frac{\left|\mu_{1}\right| a *}{m_{1}\left(1-\mathrm{e}^{-a * T}\right)}+\frac{\left|\mu_{2}\right|}{m_{1}\left(\mathrm{e}^{a * T}-1\right)}+m_{1}^{-1} M_{1}^{*}\left(\left|\mu_{0}\right|+\alpha^{-1}\left|\mu_{2}\right|+\alpha^{-1} T\|F\|_{\infty}\right)\right], \\
c_{0}=\left|\mu_{0}\right|+\alpha^{-1}\left|\mu_{2}\right|+\alpha^{-1} T\|F\|_{\infty}+\alpha^{-1} c_{0} M_{1} T, \quad F(t)=f(t, 0,0)
\end{gathered}
$$

and

$$
\left|u^{(k)}(t)\right| \leq C\left(1+\frac{1}{\varepsilon^{k}} \mathrm{e}^{-\frac{\alpha t}{\varepsilon}}\right), k=1,2, t \in[0, T],
$$

provided $a \in C^{1}[0, T]$ and $\left|\frac{\partial f}{\partial t}\right| \leq C$ for $t \in[0, T]$ and $|u| \leq c_{1},|\lambda| \leq c_{0}$.

Proof. We rewrite Equation (1.1) in form

$$
\varepsilon u^{\prime \prime}-a(t) u^{\prime}-b(t) u-\lambda c(t)-F(t)=0, t \in[0, T],
$$

where, $b(t)=\frac{\partial f}{\partial u}(t, \tilde{u}, \tilde{\lambda}), c(t)=\frac{\partial f}{\partial \lambda}(t, \tilde{u}, \tilde{\lambda}), \tilde{u}=\gamma u, \tilde{\lambda}=\gamma \lambda(0<\gamma<1)$ intermediate values.

From (2.8) for the first derivate, we have

$$
u^{\prime}(t)=\frac{\mu_{2}}{\varepsilon} \mathrm{e}^{-\frac{1}{\varepsilon} \int_{t}^{T}(t) \mathrm{d} t}-\frac{1}{\varepsilon} \int_{t}^{T} b(\tau) u(\tau) e^{-\frac{1}{\varepsilon} \tau_{t}^{\tau} \alpha(t) \mathrm{d} t} \mathrm{~d} \tau-\frac{\lambda}{\varepsilon} \int_{t}^{T} c(\tau) \mathrm{e}^{-\frac{1}{\varepsilon} \tau_{t}^{\tau} \alpha(t) \mathrm{d} t} \mathrm{~d} \tau-\frac{1}{\varepsilon} \int_{t}^{T} F(\tau) \mathrm{e}^{-\frac{1}{\varepsilon} \int_{t}^{\tau}(t) \mathrm{d} t} \mathrm{~d} \tau,
$$

from which, after using the initial condition $u^{\prime}(0)=\mu_{1}$, it follows that,

$$
\begin{aligned}
\lambda= & \frac{-\mu_{1}}{\frac{1}{\varepsilon} \int_{0}^{T} c(\tau) \mathrm{e}^{-\frac{1}{\varepsilon} \int_{0}^{T} a(t) \mathrm{d} t} \mathrm{~d} \tau}+\frac{\frac{\mu_{2}}{\varepsilon} \mathrm{e}^{-\frac{1}{\varepsilon} \int_{0}^{T} a(t) \mathrm{d} t}}{\frac{1}{\varepsilon} \int_{0}^{T} c(\tau) \mathrm{e}^{-\frac{1}{\varepsilon} \int_{0}^{T} a(t) \mathrm{d} t} \mathrm{~d} \tau} \\
& -\frac{\frac{1}{\varepsilon} \int_{0}^{T} b(\tau) u(\tau) \mathrm{e}^{-\frac{1}{\varepsilon} \int_{0}^{T} a(t) \mathrm{d} t}}{\frac{1}{\varepsilon} \int_{0}^{T} c(\tau) \mathrm{e}^{-\frac{1}{\varepsilon} \int_{0}^{T} a(t) \mathrm{d} t} \mathrm{~d} \tau}-\frac{\frac{1}{\varepsilon} \int_{0}^{T} F(\tau) \mathrm{e}^{-\frac{1}{\varepsilon} \int_{0}^{T} a(t) \mathrm{d} t}}{\frac{1}{\varepsilon} \int_{0}^{T} c(\tau) \mathrm{e}^{-\frac{-}{\varepsilon} \int_{0}^{T} a(t) \mathrm{d} t} \mathrm{~d} \tau}
\end{aligned}
$$

Applying the mean value theorem for integrals, we deduce that,

$$
\left|\frac{\frac{1}{\varepsilon} \int_{0}^{T} F(\tau) \mathrm{e}^{-\frac{1}{\varepsilon} \int_{0}^{T} a(t) \mathrm{d} t}}{\mid \frac{1}{\varepsilon} \int_{0}^{T} c(\tau) \mathrm{e}^{-\frac{1}{\varepsilon} \int_{0}^{T} \alpha(t) \mathrm{d} t} \mathrm{~d} \tau}\right| \leq \frac{\|F\|_{\infty}\left|\int_{0}^{T} \mathrm{e}^{-\frac{1}{\varepsilon} \int_{0}^{T} a(t) \mathrm{d} t}\right|}{m_{1}\left|\int_{0}^{T} \mathrm{e}^{-\frac{1}{\varepsilon} \int_{0}^{T} a(t) \mathrm{d} t} \mathrm{~d} \tau\right|} \leq m_{1}^{-1}\|F\|_{\infty}
$$

and

$$
\left|\frac{\frac{1}{\varepsilon} \int_{0}^{T} b(\tau) u(\tau) \mathrm{e}^{-\frac{1}{\varepsilon} \int_{0}^{T} a(t) \mathrm{d} t}}{\frac{1}{\varepsilon} \int_{0}^{T} c(\tau) \mathrm{e}^{-\frac{1}{\varepsilon} \int_{0}^{T} a(t) \mathrm{d} t} \mathrm{~d} \tau}\right| \leq \frac{M_{1}^{*}\|u\|_{\infty}\left|\int_{0}^{T} \mathrm{e}^{-\frac{1}{\varepsilon} \int_{0}^{T} a(t) \mathrm{d} t}\right|}{m_{1}\left|\int_{0}^{T} \mathrm{e}^{-\frac{1}{\varepsilon} \int_{0}^{T} a(t) \mathrm{d} t} \mathrm{~d} \tau\right|} \leq m_{1}^{-1} M_{1}^{*}\|u\|_{\infty}
$$


Also, for first and second terms in right side of (2.10) for $\varepsilon \leq 1$ values, we have

$$
\frac{\left|\mu_{1}\right|}{\left|\frac{1}{\varepsilon} \int_{0}^{T} c(\tau) \mathrm{e}^{-\frac{1}{\varepsilon} \int_{0}^{T} a(t) \mathrm{d} t} \mathrm{~d} \tau\right|}+\frac{\frac{\left|\mu_{2}\right|}{\varepsilon} \mathrm{e}^{-\frac{1}{\varepsilon} \int_{0}^{T} a(t) \mathrm{d} t}}{\left|\frac{1}{\varepsilon} \int_{0}^{T} c(\tau) \mathrm{e}^{-\frac{1}{\varepsilon} \int_{0}^{T} a(t) \mathrm{d} t} \mathrm{~d} \tau\right|} \leq \frac{\left|\mu_{1}\right|}{m_{1}(a *)^{-1}\left(1-\mathrm{e}^{-\frac{a * T}{\varepsilon}}\right)}+\frac{\left|\mu_{2}\right|}{m_{1} \varepsilon\left(\mathrm{e}^{\frac{\alpha T}{\varepsilon}}-1\right)} .
$$

It then follows from (2.11)-(2.13),

$$
|\lambda| \leq \frac{a *\left|\mu_{1}\right|}{m_{1}\left(1-\mathrm{e}^{-a * T}\right)}+\frac{\left|\mu_{2}\right|}{m_{1}\left(\mathrm{e}^{\alpha T}-1\right)}+m_{1}^{-1} M_{1}^{*}\|u\|_{\infty}+m_{1}^{-1}\|F\|_{\infty}
$$

Further from (2.4) by taking $\Phi(t)=\lambda c(t)+F(t)$ we get

$$
\|u\|_{\infty} \leq\left|\mu_{0}\right|+\alpha^{-1}\left|\mu_{2}\right|+\alpha^{-1} T\|F\|_{\infty}+\alpha^{-1} M_{1} T|\lambda| .
$$

The inequlities (2.14), (2.15) immediately leads to (2.5), (2.6). After taking into consideration the uniformly boundnees in $\varepsilon$ of $u(t)$ and $\lambda$, it then follows from (2.9) that,

$$
\left|u^{\prime}(t)\right| \leq \frac{\left|\mu_{2}\right|}{\varepsilon} \mathrm{e}^{-\frac{1}{\varepsilon} \int_{0}^{T} \alpha(t) \mathrm{d} t}+\frac{1}{\varepsilon} c_{1} M_{1}^{*} \int_{t}^{T} \mathrm{e}^{-\frac{1}{\varepsilon} \int^{\tau} t^{\tau}(t) \mathrm{d} t} \mathrm{~d} \tau+\frac{1}{\varepsilon} c_{0} M_{1} \int_{t}^{T} \mathrm{e}^{-\frac{1}{\varepsilon} \int_{t}^{\tau} \alpha(t) \mathrm{d} t} \mathrm{~d} \tau+\frac{1}{\varepsilon}\|F\|_{\infty} \int_{t}^{T} \mathrm{e}^{-\frac{1}{\varepsilon} \int_{t}^{\tau} \alpha(t) \mathrm{d} t} \mathrm{~d} \tau,
$$

which proves (2.7) for $k=1$. To obtain (2.7) for $k=2$, first from (1.1) we have

$$
\left|u^{\prime \prime}(T)\right| \leq \frac{a(T) u^{\prime}(T)+F(T, u(T), \lambda)}{\varepsilon}
$$

from which after taking into consideration here $u^{\prime}(T)=\frac{\mu_{2}}{\varepsilon}$ and (2.5) we obtain

$$
\left|u^{\prime \prime}(T)\right| \leq \frac{C}{\varepsilon^{2}} .
$$

Next, differentiation (1.1) gives

$$
\begin{gathered}
\varepsilon v^{\prime \prime}-a(t) v^{\prime}-Q(t)=0, \quad 0<t<T, \\
v^{\prime}(T)=O\left(\varepsilon^{-2}\right)
\end{gathered}
$$

with

$$
\begin{gathered}
v(t)=u^{\prime}(t), \\
Q(t)=a^{\prime}(t) v(t)+\frac{\partial f}{\partial t}(t, u(t), \lambda)+\frac{\partial f}{\partial u}(t, u(t), \lambda),
\end{gathered}
$$

and due to our assumptions clearly,

$$
|Q(t)| \leq C\left(1+\frac{1}{\varepsilon} \mathrm{e}^{-\frac{\alpha(T-t)}{\varepsilon}}\right) .
$$

Consequently, from (2.17), (2.18) we have

$$
\left|v^{\prime}(t)\right| \leq \frac{C}{\varepsilon^{2}} \mathrm{e}^{-\frac{\alpha(T-t)}{\varepsilon}}+\frac{1}{\varepsilon} \int_{t}^{T}|Q(t)| \mathrm{e}^{-\frac{\alpha(T-t)}{\varepsilon}}=\frac{C}{\mathcal{E}^{2}} \mathrm{e}^{-\frac{\alpha(T-t)}{\varepsilon}}+C\left(1+\frac{1}{\varepsilon}\right) \int_{t}^{T} \mathrm{e}^{-\frac{\alpha(T-t)}{\varepsilon}}
$$

which proves (2.7) for $k=2$.

Example. Consider the following parameterized singular perturbation problem:

$$
\varepsilon u^{\prime \prime}-a(t) u^{\prime}+\lambda+\tanh (1-t+\lambda)=f(t), 0<t<1,
$$




$$
u(0)=\mu_{0}, \quad u^{\prime}(0)=\mu_{1}, \quad u^{\prime}(T)=\frac{\mu_{2}}{\varepsilon}
$$

with

$$
\mu_{1}=\frac{2}{2+\varepsilon}\left[\frac{\mathrm{e}+1+\varepsilon}{\varepsilon\left(\mathrm{e}^{\frac{2}{\varepsilon}}-1\right)}-\frac{\mathrm{e}}{2}\right], \mu_{2}=\frac{2 \mathrm{e}+2+\varepsilon\left(1+\mathrm{e}^{-\frac{2}{\varepsilon}}\right)}{1-\mathrm{e}^{-\frac{2}{\varepsilon}}}
$$

and $f(t)$ selected so that the solution is

$$
u(t)=\gamma_{1}+\gamma_{2} \mathrm{e}^{-\frac{2}{\varepsilon}(1-t)}+\frac{1}{2+\varepsilon} \mathrm{e}^{1-t}, \lambda=0.5
$$

where,

$$
\gamma_{1}=-\frac{\mathrm{e}+(1+\varepsilon) \mathrm{e}^{-\frac{2}{\varepsilon}}}{(2+\varepsilon)\left(1-\mathrm{e}^{-\frac{2}{\varepsilon}}\right)}, \quad \gamma_{2}=\frac{1+\mathrm{e}+\varepsilon}{(2+\varepsilon)\left(1-\mathrm{e}^{-\frac{2}{\varepsilon}}\right)} .
$$

First and second derivatives have the form

$$
u(t)=\left(\frac{2}{\varepsilon}\right)^{k} \gamma_{2} \mathrm{e}^{-\frac{2}{\varepsilon}(1-t)}+\frac{1}{2+\varepsilon} \mathrm{e}^{1-t}, \quad k=1,2 .
$$

Therefore, we observe here the accordance in our theoretical results described above.

\section{References}

[1] Fěckan, M. (1994) Parametrized Singularly Perturbed Boundary Value Problems. Journal of Mathematical Analysis and Applications, 188, 426-435. http://dx.doi.org/10.1006/jmaa.1994.1436

[2] Goma, I.A. (1977) Method of Successive Approximations in a Two-Point Boundary Problem with Parameter. Ukrainian Mathematical Journal, 29, 594-599. http://dx.doi.org/10.1007/BF01085968

[3] Jankowski, T. and Lakshmikantham, V. (1997) Monotone Iterations for Differential Equations with a Parameter. Journal of Applied Mathematics and Stochastic Analysis, 10, 273-278. http://dx.doi.org/10.1155/S1048953397000348

[4] Jankowski, T. (1999) Generalization of the Method of Quasilinearization for Differential Problems with a Parameter. Dynamic Systems and Applications, 8, 53-72.

[5] Jankowski, T. (2001) Monotone Iterations for Differential Problems. Mathematical Notes (Miskolc), 2, 31-38.

[6] Pomentale, T. (1976) A Constructive Theorem of Existence and Uniqueness for the Problem, $y^{\prime}=f(x, y, \lambda)$, $y(a)=\alpha, y(b)=\beta$. Zeitschrift für Angewandte Mathematik und Mechanik, 56, 387-388.

http://dx.doi.org/10.1002/zamm.19760560806

[7] Rontó, M. and Csikos-Marinets, T. (2000) On the Investigation of Some Non-Linear Boundary Value Problems with Parameters. Mathematical Notes (Miskolc), 1, 157-166.

[8] Rontó, M. (2000) On Non-Linear Boundary Value Problems Containing Parameters. Archivum Mathematicum, 36, 585-593.

[9] Staněk, S. (1997) Nonlinear Boundary Value Problem for Second Order Differential Equations Depending on a Parameter. Mathematica Slovaca, 47, 439-449.

[10] Zhang, P. (2011) Existence of Positive Solutions for Nonlocal Second-Order Boundary Value Problem with Variable Parameter in Banach Spaces. Fixed Point Theory and Applications, 43, 1687-1812. http://dx.doi.org/10.1186/1687-1812-2011-43

[11] Amiraliyev, G.M., Kudu, M. and Duru, H. (2004) Finite-difference Method for Parameterized Singularly Perturbed Problem. Journal of Applied Mathematics, 3, 191-199.

[12] Amiraliyev, G.M., Kudu, M. and Duru, H. (2006) Uniform Difference Method for A Parameterized Singular Perturba- 
tion Problem. Applied Mathematics and Computation, 175, 89-100. http://dx.doi.org/10.1016/j.amc.2005.07.068

[13] Amiraliyeva, I.G. and Amiraliyev, G.M. (2009) Uniform Difference Method for Parameterized Singularly Perturbed Delay Differential Equations. Numerical Algorithms, 52, 509-552. http://dx.doi.org/10.1007/s11075-009-9295-y

[14] Turkyilmazoglu, M. (2011) Analytic Approximate Solutions of Parameterized Unperturbed and Singularly Perturbed Boundary Value Problems. Applied Mathematical Modelling, 35, 3879-3886. http://dx.doi.org/10.1016/j.apm.2011.02.011

[15] Farrel, P.A., Hegarty, A.F., Miller, J.J.H., O’Riordan, E. and Shishkin, G.I. (2000) Robust Computational Techniques for Boundary Layers. Chapman Hall/CRC, New York.

[16] Nayfeh, A.H. (1981) Introduction to Perturbation Techniques. John Wiley \& Sons, New York.

[17] O’Malley Jr., R.E. (1991) Singular Perturbation Methods for Ordinary Differential Equations. Springer-Verlag, New York.

[18] Roos, H.G., Stynes, M. and Tobiska, L. (2008) Robust Numerical Methods for Singularly Perturbed Differential Equations, Convection Diffusion and Flow Problems. Springer-Verlag, Berlin, Heidelberg. 